\title{
AN ELEMENTARY PROOF OF SCHMÜDGEN'S THEOREM ON THE MOMENT PROBLEM OF CLOSED SEMI-ALGEBRAIC SETS
}

\author{
TIM NETZER
}

(Communicated by Joseph A. Ball)

\begin{abstract}
We prove the main result from Schmüdgen's 2003 article in a more elementary way. The result states that the question of whether a finitely generated preordering has the so-called strong moment property can be reduced to the same question for preorderings corresponding to fiber sets of bounded polynomials.
\end{abstract}

\section{INTRODUCTION}

Preorderings in the ring of real polynomials are of great importance in Real Algebra and Real Algebraic Geometry. They correspond to semi-algebraic sets in a similar fashion as ideals correspond to algebraic sets.

Starting with Hilbert's question of whether every nonnegative real polynomial in several variables is a sum of squares of real rational functions, many questions have arisen in this field and many interesting results are known. Different kinds of Positiv- and Nullstellensätze give representations of polynomials with certain properties on semi-algebraic sets. For example, for a compact basic closed semialgebraic set, every strictly positive polynomial belongs to the corresponding finitely generated preordering (this is the main result in [S2]). If the set is not compact, then this result fails in general. See for example [PD] for a thorough treatment of the field.

Another important question concerns the so-called moment problem. When is it true that a linear form on the polynomial ring which is nonnegative on a finitely generated preordering is integration with respect to a measure? This leads to the problem of determining the closure of the preordering with respect to the finest locally convex topology on the vector space of polynomials. Indeed, if this closure consists of all polynomials which are nonnegative on the semi-algebraic set, then, by Haviland's Theorem (Theorem 2.1), every linear form nonnegative on the preordering is given by a measure on the corresponding semi-algebraic set. We say that the preordering has the strong moment property in this case.

In his 2003 article [S3], Schmüdgen proves a very strong criterion for a preordering to have this property. Indeed, if every preordering from a certain family of

Received by the editors July 26, 2006.

2000 Mathematics Subject Classification. Primary 44A60, 14P10.

Key words and phrases. Moment problem, real algebraic geometry. 
preorderings constructed from the original one has the property, then the original preordering has it as well. This is in fact a necessary and sufficient condition. Geometrically, the constructed preorderings correspond to lower dimensional semialgebraic sets, namely fiber sets of bounded polynomials. As the low-dimensional case is often better understood (see for example [KM] and [KMS]), the Schmüdgen criterion can be applied successfully in many cases.

The proof in [S3] uses deep results from functional analysis. A direct integral decomposition of a GNS representation is used to decompose a linear form on the polynomial ring into an integral of linear forms. The applied methods are from [S1] and $[D]$. In the present work we give a more elementary proof of the theorem. We also decompose a linear form into an integral of linear forms. These linear forms are constructed from functions emerging from the Radon-Nikodym Theorem.

\section{Notation AND PRELIMinaries}

For $n \in \mathbb{N}$ consider the real polynomial ring $\mathbb{R}\left[X_{1}, \ldots, X_{n}\right]$, which we will denote by $\mathbb{R}[X]$ in the following. Later we will also use the polynomial ring $\mathbb{R}[Y]=$ $\mathbb{R}\left[Y_{1}, \ldots, Y_{s}\right]$ for some $s \in \mathbb{N}$. To distinguish between elements, we will use capitals for elements from $\mathbb{R}[Y]$ and lowercase letters for elements from $\mathbb{R}[X]$.

For finitely many polynomials $f_{1}, \ldots, f_{r} \in \mathbb{R}[X]$ we write $T=T\left(f_{1}, \ldots, f_{r}\right)$ for the preordering generated by these polynomials, which consists of all finite sums of elements of the form

$$
\sigma f_{1}^{e_{1}} \cdots f_{r}^{e_{r}}
$$

where $\sigma$ is a sum of squares of real polynomials and all $e_{i} \in\{0,1\}$. On the geometric side, we have the so-called basic closed semi-algebraic set $W=W\left(f_{1}, \ldots, f_{r}\right)$ corresponding to $f_{1}, \ldots, f_{r}$, defined as

$$
W=\left\{x \in \mathbb{R}^{n} \mid f_{1}(x) \geq 0, \ldots, f_{r}(x) \geq 0\right\} .
$$

We then consider the saturation of $T$, denoted by $T^{\mathrm{sat}}$, consisting of all real polynomials nonnegative on $W$. Note that $T^{\text {sat }}$ depends only on $W$, whereas $T$ can be different for different sets of generators defining the same set $W$.

Obviously $T \subseteq T^{\text {sat }}$ and $T^{\text {sat }}$ is again a preordering, i.e. $T^{\text {sat }}$ is closed under addition and multiplication and contains all squares of polynomials. The relation between $T$ and $T^{\text {sat }}$ is an important object of study in Real Algebraic Geometry.

As $\mathbb{R}[X]$ is a real vector space and $T$ is a convex cone, we define

$$
T^{\vee}:=\{L: \mathbb{R}[X] \rightarrow \mathbb{R} \text { linear } \mid L(T) \subseteq[0, \infty) \text { and } L(1)=1\}
$$

the algebraic dual cone of $T$. As explained in $[\mathrm{KM}], L(1)>0$ holds for all $L \neq 0$ which are nonnegative on $T$. Therefore the condition $L(1)=1$ in the definition of $T^{\vee}$ can be ensured for those forms by scaling with a positive real and it does not affect any of the following considerations.

We define the double dual cone

$$
T^{\vee \vee}:=\left\{p \in \mathbb{R}[X] \mid L(p) \geq 0 \text { for all } L \in T^{\vee}\right\} .
$$

$T^{\vee \vee}$ is the closure of $T$ with respect to the finest locally convex topology on the vector space $\mathbb{R}[X]$. It is again a preordering and we have

$$
T \subseteq T^{\vee \vee} \subseteq T^{\mathrm{sat}}
$$


the first inclusion being obvious, the second one coming from the fact that evaluation in $x \in W$ defines an element from $T^{\vee}$. See [KM] and [KMS] for a more thorough discussion.

Now one is interested in the relation between $T^{\vee \vee}$ and $T^{\text {sat }}$. In particular, one wants to know whether $T^{\vee \vee}=T^{\text {sat }}$ holds. Following the notation in [S3], we say that the preordering $T$ has the strong moment property (SMP) in this case. The importance of this notion is obvious from the following classical theorem by Haviland $[\mathrm{H}]$.

Theorem 2.1 (Haviland). Let $K$ be a closed subset of $\mathbb{R}^{n}$ and $L: \mathbb{R}[X] \rightarrow \mathbb{R}$ be a linear form. Then $L$ is given by a positive Borel measure $\nu$ on $K$ (i.e. $L(f)=$ $\int_{K} f d \nu$ for all $\left.f \in \mathbb{R}[X]\right)$ if and only if $L(f) \geq 0$ for all $f$ nonnegative on $K$.

So if $T$ has SMP, then every $L \in T^{\vee}$ is nonnegative on $T^{\text {sat }}$ and is given by a positive Borel measure on $W$, by Haviland's Theorem. As it is much easier to check nonnegativity on $T$ than on $T^{\text {sat }}$, SMP is a useful property.

Schmüdgen proved in [S2] that $T$ has SMP whenever $W$ is compact, independent of the choice of generators. Indeed, he proved a bit more without bringing it up explicitly. Denote by $\mathcal{B}_{W}$ the ring of all polynomials which are bounded on $W$. Then, even if $W$ is not compact we have:

Theorem 2.2 (Schmüdgen). $\mathcal{B}_{W} \cap T^{\mathrm{sat}} \subseteq T^{\vee \vee}$.

If $W$ is compact, then every polynomial is bounded on $W$ and therefore the theorem shows $T^{\vee \vee}=T^{\mathrm{sat}}$.

As this result is not stated explicitly in [S2], we give a short sketch of the proof. Proof. For every $b \in \mathcal{B}_{W}$ and every $L \in T^{\vee}$, we have

$$
L\left(b^{2}\right) \leq\|b\|_{\infty}^{2},
$$

where $\|b\|_{\infty}$ denotes the supremum of $b$ on $W$. This is shown in the proof of Theorem 1 in [S2] or in the proof of Proposition 2 in [S3]. It uses the Positivstellensatz and the one-dimensional Hamburger moment problem.

So, in addition, if $b$ is nonnegative on $W$ for any $\delta>\|b\|_{\infty}$, we have

$$
-\delta \leq b-\delta<0
$$

on $W$, and so

$$
L\left((b-\delta)^{2}\right) \leq \delta^{2},
$$

using (1). This yields $0 \leq L\left(b^{2}\right) \leq 2 \delta L(b)$, so $L(b) \geq 0$ since $\delta>0$. This shows $b \in T^{\vee \vee}$.

The noncompact case was investigated by Schmüdgen in [S3]. He reduced the question of whether $T$ has SMP to the same question for preorderings corresponding to lower-dimensional semi-algebraic sets, namely fibers of polynomials bounded on $W$.

\section{MAIN THEOREM}

For the present section fix $f_{1}, \ldots, f_{r} \in \mathbb{R}[X]$ and consider $T, W$ and $\mathcal{B}_{W}$ as in Section 2. In addition, we fix bounded polynomials $h_{1}, \ldots, h_{s} \in \mathcal{B}_{W}$. We will often write $h$ in the following if we refer to the $s$-tuple $h_{1}, \ldots, h_{s}$. The set

$$
h(W):=\left\{\left(h_{1}(x), \ldots, h_{s}(x)\right) \mid x \in W\right\}
$$


is a bounded subset of $\mathbb{R}^{s}$, and its closure with respect to the usual topology is denoted by $\overline{h(W)}$.

For any $\lambda=\left(\lambda_{1}, \ldots, \lambda_{s}\right) \in \mathbb{R}^{s}$ we consider the preordering

$$
T_{\lambda}:=T+I_{\lambda},
$$

where $I_{\lambda}:=\left(h_{1}-\lambda_{1}, \ldots, h_{s}-\lambda_{s}\right)$ is the ideal generated by the $h_{i}-\lambda_{i} . T_{\lambda}$ is again a finitely generated preordering, as every polynomial is a difference of two squares of polynomials, indeed generated by $f_{1}, \ldots, f_{r}, \pm\left(h_{1}-\lambda_{1}\right), \ldots, \pm\left(h_{s}-\lambda_{s}\right)$. This shows that the basic closed semi-algebraic set $W_{\lambda}$ corresponding to $T_{\lambda}$ is just $W$ intersected with the zero set of $I_{\lambda}$.

Now Schmüdgen proved the remarkable theorem that if $T_{\lambda}$ has SMP for all $\lambda \in h(W)$, then so does $T$ ([S3], see Corollary 3.3 below). He used direct integral decompositions of $*$-represenations of $*$-algebras. Indeed, he proved the following slightly stronger theorem, which we will prove here in a more elementary way.

\section{Theorem 3.1.}

$$
T^{\vee \vee}=\bigcap_{\lambda \in h(W)} T_{\lambda}^{\vee \vee}
$$

Before we begin with the proof, we explain some constructions that we will use. We fix $L \in T^{\vee}$ and define linear forms $L_{p}$ on $\mathbb{R}[Y]=\mathbb{R}\left[Y_{1}, \ldots, Y_{s}\right]$ for any $p \in \mathbb{R}[X]$ in the following way:

$$
L_{p}: \mathbb{R}[Y] \rightarrow \mathbb{R} ; \quad F \mapsto L(F(h) p) .
$$

Here, $F(h)$ is an abbreviation for $F\left(h_{1}, \ldots, h_{s}\right)$, where $F \in \mathbb{R}[Y]$. Since $p=$ $\left(\frac{p+1}{2}\right)^{2}-\left(\frac{p-1}{2}\right)^{2}$ for any $p \in \mathbb{R}[X]$, we have

$$
L_{p}=L_{\left(\frac{p+1}{2}\right)^{2}}-L_{\left(\frac{p-1}{2}\right)^{2}} .
$$

Now for $p \in \mathbb{R}[X]$, the linear form $L_{p^{2}}$ fulfills the condition of Haviland's theorem. Indeed, if $F \in \mathbb{R}[Y]$ is nonnegative on $\overline{h(W)}$, then $F(h)$ is nonnegative on $W$. As all $h_{i}$ are bounded on $W$, so is $F(h)$. By Theorem 2.2 we get $F(h) \in T^{\vee \vee}$. Being the closure of $T$ in the finest locally convex topology on $\mathbb{R}[X], T^{\vee \vee}$ is again a preordering, and so $F(h) p^{2} \in T^{\vee \vee}$, which yields $0 \leq L\left(F(h) p^{2}\right)=L_{p^{2}}(F)$.

Applying Theorem 2.1, there is a positive Borel measure $\nu_{p}$ on $\overline{h(W)}$ with

$$
L_{p^{2}}(F)=L\left(F(h) p^{2}\right)=\int_{\frac{h(W)}{h}} F d \nu_{p} \quad \text { for all } F \in \mathbb{R}[Y] .
$$

These measures are obviously finite and therefore regular. As all considered measures are defined on $\overline{h(W)}$, we omit the subscripts under the integral signs from now on.

The following lemma is an important ingredient in the proof of Theorem 3.1.

Lemma 3.2. For all $p \in \mathbb{R}[X]$,

$$
\nu_{p} \ll \nu_{1},
$$

that is, every $\nu_{1}$-null set is also a $\nu_{p}$-null set.

Proof. Take $p \in \mathbb{R}[X]$. For any $F \in \mathbb{R}[Y]$ and any $t \in \mathbb{R}$, we have

$$
0 \leq L\left(\left(F(h)+t p^{2}\right)^{2}\right)=L\left(F(h)^{2}\right)+2 t L\left(F(h) p^{2}\right)+t^{2} L\left(p^{4}\right),
$$

which implies

$$
4 L\left(F(h) p^{2}\right)^{2}-4 L\left(F(h)^{2}\right) L\left(p^{4}\right) \leq 0
$$


and so

$$
L\left(F(h) p^{2}\right)^{2} \leq L\left(F(h)^{2}\right) L\left(p^{4}\right) .
$$

Now let $A \subseteq \overline{h(W)}$ be a $\nu_{1}$-null set.

We choose a sequence $\left(f_{n}\right)_{n \in \mathbb{N}}$ of continuous functions on $\overline{h(W)}$, which take on values in the interval $[0,1]$ and which converge pointwise, except on a set $N$ which is a $\nu_{1}$ - and a $\nu_{p}$-null set, to the characteristic function $\chi_{A}$ of $A$. This can be done using the fact that both measures are regular and by applying Urysohn's Lemma.

Due to the Theorem of Majorized Convergence, we get

$$
\int f_{n} d \nu_{1} \longrightarrow \int \chi_{A} d \nu_{1}=0
$$

Now take a sequence of polynomials $\left(Q_{n}\right)_{n \in \mathbb{N}} \subset \mathbb{R}[Y]$ such that

$$
\left\|Q_{n}-\sqrt{f_{n}}\right\|_{\infty} \stackrel{n \rightarrow \infty}{\longrightarrow} 0
$$

where $\|\cdot\|_{\infty}$ denotes the supremum on the compact set $\overline{h(W)}$. So

$$
\left|\int Q_{n}^{2} d \nu_{1}-\int f_{n} d \nu_{1}\right| \leq\left\|Q_{n}^{2}-f_{n}\right\|_{\infty} \cdot \nu_{1}(\overline{h(W)}) \stackrel{n \rightarrow \infty}{\longrightarrow} 0 .
$$

Further we get

$$
\begin{aligned}
\left(\int Q_{n} d \nu_{p}\right)^{2} & =L\left(Q_{n}(h) p^{2}\right)^{2} \\
& \leq L\left(Q_{n}(h)^{2}\right) L\left(p^{4}\right) \\
& =L\left(p^{4}\right) \int Q_{n}^{2} d \nu_{1},
\end{aligned}
$$

where the inequality uses (3). So (4) combined with (6) yields

$$
\int Q_{n} d \nu_{p} \stackrel{n \rightarrow \infty}{\longrightarrow} 0
$$

As the sequence $\left(\sqrt{f_{n}}\right)_{n \in \mathbb{N}}$ obviously also converges pointwise except on $N$ to $\chi_{A}$, so does the sequence $\left(Q_{n}\right)_{n \in \mathbb{N}}$ by (5). As $N$ is also a $\nu_{p}$-null set, again by the Theorem of Majorized Convergence,

$$
\int Q_{n} d \nu_{p} \longrightarrow \int \chi_{A} d \nu_{p}=\nu_{p}(A)
$$

So $\nu_{p}(A)=0$, which was to be shown.

Lemma 3.2 allows us to apply the Radon-Nikodym Theorem. For every $p \in \mathbb{R}[X]$ we get a $\nu_{1}$-integrable function

$$
\Phi_{p}: \overline{h(W)} \rightarrow[0, \infty)
$$

such that

$$
L_{p^{2}}(F)=\int F d \nu_{p}=\int F \Phi_{p} d \nu_{1} \quad \text { for all } F \in \mathbb{R}[Y] .
$$

If we define $\theta_{p}:=\Phi_{\frac{p+1}{2}}-\Phi_{\frac{p-1}{2}}$ for $p \in \mathbb{R}[X]$, then all $\theta_{p}$ are $\nu_{1}$-integrable and

$$
L_{p}(F)=L(F(h) p)=\int F \theta_{p} d \nu_{1}
$$

holds for every $F \in \mathbb{R}[Y]$ by (2). 
Before we start with the proof of Theorem 3.1, we look at some of the properties of the $\theta_{p}$.

For $g_{1}, g_{2} \in \mathbb{R}[X], t_{1}, t_{2} \in \mathbb{R}$, and any $F \in \mathbb{R}[Y]$, we have

$$
\begin{aligned}
\int F \theta_{t_{1} g_{1}+t_{2} g_{2}} d \nu_{1} & =L\left(F(h)\left(t_{1} g_{1}+t_{2} g_{2}\right)\right) \\
& =L\left(F(h) t_{1} g_{1}\right)+L\left(F(h) t_{2} g_{2}\right) \\
& =\int F t_{1} \theta_{g_{1}} d \nu_{1}+\int F t_{2} \theta_{g_{2}} d \nu_{1} \\
& =\int F\left(t_{1} \theta_{g_{1}}+t_{2} \theta_{g_{2}}\right) d \nu_{1} .
\end{aligned}
$$

By Lemma 4.1 from the Appendix, this implies

$$
\theta_{t_{1} g_{1}+t_{2} g_{2}}=t_{1} \theta_{g_{1}}+t_{2} \theta_{g_{2}}
$$

except on a $\nu_{1}$-null set which depends on $g_{1}, g_{2}, t_{1}, t_{2}$.

Further, for any $Q \in \mathbb{R}[Y]$ and $t \in T$,

$$
0 \leq L\left(Q(h)^{2} t\right)=\int Q^{2} \theta_{t} d \nu_{1}
$$

holds, so again by Lemma 4.1,

$$
\theta_{t} \geq 0
$$

except on a $\nu_{1}$-null set depending on $t$.

Last, for $p \in \mathbb{R}[X], Q \in \mathbb{R}[Y]$ and any $F \in \mathbb{R}[Y]$, we have

$$
\begin{aligned}
\int F \theta_{Q(h) p} d \nu_{1} & =L(F(h) Q(h) p) \\
& =\int F Q \theta_{p} d \nu_{1} .
\end{aligned}
$$

So by Lemma 4.1,

$$
\theta_{Q(h) p}=Q \cdot \theta_{p}
$$

except on a $\nu_{1}$-null set depending on $p$ and $Q$.

With all these constructions in mind, we prove the main theorem.

Proof of Theorem 3.1. One of the inclusions is obvious, for the other one fix $f \in$ $\bigcap_{\lambda \in h(W)} T_{\lambda}^{\vee \vee}$. Take $L \in T^{\vee}$. We have to show $L(f) \geq 0$.

Define

$$
A:=\mathbb{Q}\left[X_{1}, \ldots, X_{n}, f_{1}, \ldots, f_{r}, h_{1}, \ldots, h_{s}, f\right] .
$$

(Remember that the $f_{i}$ are the polynomials defining $W$ and $T$, whereas the $h_{i}$ are the bounded polynomials we fixed.) Any $a \in A$ can be written as a real polynomial in the form

$$
a=\sum a_{\alpha} X^{\alpha}
$$

where the $a_{\alpha}$ are real, $\alpha=\left(\alpha_{1}, \ldots, \alpha_{n}\right) \in \mathbb{N}^{n}, X^{\alpha}:=X_{1}^{\alpha_{1}} \cdots X_{n}^{\alpha_{n}}$, and the sum is finite. We will use this representation for $a$ in the following.

From $L$ we construct all the functions $\theta_{p}$ introduced above. Using (8), (9) and (10), we find a single $\nu_{1}$-null set $N \subseteq \overline{h(W)}$, such that the following conditions are 
true for all $\lambda \notin N$ :

$$
\text { For all } a \in A \text { we have } \theta_{a}(\lambda)=\sum a_{\alpha} \theta_{X^{\alpha}}(\lambda) \text {. }
$$

For all $t \in A \cap T$ we have $\theta_{t}(\lambda) \geq 0$.

$$
\text { For all } p \in \mathbb{Q}[X], Q \in \mathbb{Q}[Y] \text { we have } \theta_{Q(h) p}(\lambda)=Q(\lambda) \theta_{p}(\lambda) \text {. }
$$

As $A$ is countable, we can indeed ensure all this with one single null set $N$.

Now for $\lambda \in \overline{h(W)} \backslash N$ we define a linear form $L_{\lambda}$ on $\mathbb{R}[X]$ by

$$
L_{\lambda}\left(X^{\alpha}\right):=\theta_{X^{\alpha}}(\lambda) \quad \text { for all } \alpha \in \mathbb{N}^{n} .
$$

These linear forms fulfill

$$
L_{\lambda}(a)=\theta_{a}(\lambda)
$$

for all $a \in A$ by (11). So for any $t \in A \cap T$ we have

$$
L_{\lambda}(t)=\theta_{t}(\lambda) \geq 0,
$$

using (12). As we can approximate every element from $T$ coefficientwise by a sequence of elements from $A \cap T$ of bounded degree,

$$
L_{\lambda}(T) \subseteq[0, \infty)
$$

holds.

Using (14) and (13), for any $p \in \mathbb{Q}[X], \xi \in \mathbb{Q}$ and $i \in\{1, \ldots, s\}$,

$$
\begin{aligned}
L_{\lambda}\left(\left(h_{i}-\xi\right) p\right) & =\theta_{\left(h_{i}-\xi\right) p}(\lambda) \\
& =\left(Y_{i}-\xi\right)(\lambda) \cdot \theta_{p}(\lambda) \\
& =\left(\lambda_{i}-\xi\right) \theta_{p}(\lambda) .
\end{aligned}
$$

By approximating first $\lambda_{i}$ by elements $\xi$ from $\mathbb{Q}$ and then arbitrary $p \in \mathbb{R}[X]$ by elements from $\mathbb{Q}[X]$ of bounded degree, this shows

$$
L_{\lambda}\left(I_{\lambda}\right)=\{0\} .
$$

Combined with (15) this implies

$$
L_{\lambda}\left(T_{\lambda}\right) \subseteq[0, \infty) .
$$

For $\lambda \in N$ we define $L_{\lambda} \equiv 0$. As $f$ is in $A$, we get

$$
\int_{\overline{h(W)}} L_{\lambda}(f) d \nu_{1}(\lambda)=\int_{\frac{h(W)}{\nu^{\prime}}} \theta_{f}(\lambda) d \nu_{1}(\lambda)=L_{f}(1)=L(f),
$$

using (14) and $\nu_{1}(N)=0$ for the first equality.

Now the rest is straighforward. For any $\lambda \in N$ we have $L_{\lambda}(f)=0$. For $\lambda \in$ $h(W) \backslash N$ we get $L_{\lambda}(f) \geq 0$ from the assumption, as $L_{\lambda}$ is either the zero form or an element in $T_{\lambda}^{\vee}$ by (16), maybe after scaling with a positive real. Finally, if $\lambda \in \overline{h(W)} \backslash h(W)$, this means that the semi-algebraic set $W_{\lambda}$ corresponding to $T_{\lambda}$ is empty. It is a corollary of the Positivstellensatz that $T_{\lambda}$ equals $\mathbb{R}[X]$ in this case (see for example [PD], Remark 4.2.13). So again by $(16), L_{\lambda} \equiv 0$. Thus

$$
L_{\lambda}(f) \geq 0
$$

holds for all $\lambda \in \overline{h(W)}$, so (17) yields $L(f) \geq 0$. This completes the proof.

The most important corollary of Theorem 3.1 is the following, which is Theorem 1 in $[\mathrm{S} 3]$.

Corollary 3.3. If $T_{\lambda}$ has SMP for all $\lambda \in h(W)$, then so does $T$. 
Proof. Obviously $T^{\text {sat }}=\bigcap_{\lambda \in h(W)} T_{\lambda}^{\text {sat }}$. So by Theorem 3.1,

$$
T^{\vee \vee}=\bigcap_{\lambda \in h(W)} T_{\lambda}^{\vee \vee}=\bigcap_{\lambda \in h(W)} T_{\lambda}^{\mathrm{sat}}=T^{\mathrm{sat}} .
$$

The conditions in Corollary 3.3 are also necessary. If $T$ has SMP, then so do all the $T_{\lambda}$. Indeed, for any ideal $I, T+I$ has SMP. This is Proposition 4.8 in [SCH].

There are a number of interesting examples in [S3] illustrating the use of Corollary 3.3. So we add just one more.

Example 3.4. As in [KMS], we consider the preordering

$$
T=T(X, Y, 1-X Y) \subseteq \mathbb{R}[X, Y] .
$$

The polynomial $X Y$ is bounded on $W$, all the $T_{\lambda}$ have SMP, and so does $T$.

If we change the set of defining polynomials suitably, e.g. consider

$$
T^{\prime}:=T\left(X, Y^{n}, 1-X Y\right)
$$

for some odd $n \geq 3$, the semi-algebraic set stays the same. But $T^{\prime}$ does not have SMP any more. Indeed, $Y$ is nonnegative on $W$. If $Y$ was in $T^{\prime \vee \vee}$, then by evaluating in $X=0$, we get $Y \in T\left(Y^{n}\right)^{\vee} \subseteq \mathbb{R}[Y]$. By [KM], Theorem 3.5, $T\left(Y^{n}\right)^{\vee \vee}=T\left(Y^{n}\right)$ and one checks that this preordering does not contain $Y$, a contradiction.

On the other hand, if we change the defining polynomials to

$$
\tilde{T}:=T\left(X, Y,(1-X Y)^{n}\right)
$$

for some odd $n \geq 3, \tilde{T}$ has SMP. This can again be obtained by Corollary 3.3, just as for the first preordering $T$.

Corollary 3.3 yields a general easy result about changing the defining polynomials. If $T$, defined by $f_{1}, \ldots, f_{r}$, has SMP and for example $f_{1}$ is bounded on $W$, then replacing $f_{1}$ by some odd power of $f_{1}$ preserves SMP. Indeed $T+\left(f_{1}-\lambda\right)$ has SMP for all $\lambda \in f_{1}(W)$ by the result mentioned in [SCH]. As all those $\lambda$ are nonnegative, $T\left(f_{1}^{n}, f_{2}, \ldots, f_{r}\right)+\left(f_{1}-\lambda\right)$ contains $f_{1}$ and is therefore equal to $T+\left(f_{1}-\lambda\right)$. So Corollary 3.3 also shows that $T\left(f_{1}^{n}, f_{2}, \ldots, f_{r}\right)$ has SMP.

\section{ApPEndix}

Lemma 4.1. Let $K$ be a compact subset of $\mathbb{R}^{s}$ and $\nu$ be a regular Borel measure on $K$. Let $h: K \rightarrow \mathbb{R}$ be $\nu$-integrable and suppose

$$
\int_{K} F^{2} h d \nu \geq 0 \quad \text { for all } F \in \mathbb{R}\left[Y_{1}, \ldots, Y_{s}\right] \text {. }
$$

Then there is a $\nu$-null set $N \subseteq K$, such that $h \geq 0$ except on $N$.

Proof. For $n=1,2, \ldots$, define

$$
A_{n}:=\left\{x \in K \mid-\frac{1}{n-1}<h(x) \leq-\frac{1}{n}\right\},
$$

where $\frac{1}{0}:=\infty$. Suppose $\nu\left(A_{n}\right)>0$ for some $n$ and let $\chi$ be the characteristic function of $A_{n}$. Then

$$
\int \chi h d \nu \leq-\frac{1}{n} \nu\left(A_{n}\right)<0 .
$$

Choose a sequence of continuous functions $\left(f_{n}\right)_{n \in \mathbb{N}}$ on $K$ which takes on values in $[0,1]$, such that $\int f_{n} h d \nu \stackrel{n \rightarrow \infty}{\longrightarrow} \int \chi h d \nu$. This can be done, using the regularity of 
$\nu$ as well as Urysohn's Lemma and the Theorem of Majorized Convergence. Now take $P_{n} \in \mathbb{R}[Y]$ such that $\left\|P_{n}^{2}-f_{n}\right\|_{\infty, K} \stackrel{n \rightarrow \infty}{\longrightarrow} 0$. As

$$
\left|\int P_{n}^{2} h d \nu-\int f_{n} h d \nu\right| \leq\left\|P_{n}^{2}-f_{n}\right\|_{\infty, K} \cdot \int|h| d \nu,
$$

we get $\int P_{n}^{2} h d \nu \longrightarrow \int \chi h d \nu<0$, a contradiction.

So $\nu\left(A_{n}\right)=0$ for all $n$, so $\nu\left(\bigcup_{n \in \mathbb{N}} A_{n}\right)=0$, which proves the result.

\section{ACKNOWLEDGMENTS}

I would like to thank Dr. Markus Schweighofer and Prof. Robert Denk from Konstanz University for many interesting and helpful discussions on the topic of this work.

Financial support by the Studienstiftung des Deutschen Volkes is greatfully acknowledged.

During the work on this article, Prof. Murray Marshall from the University of Saskatchewan found a similar approach to the problem independently.

\section{REFERENCES}

[D] J. Dixmier, Les algèbres d'opérateurs dans l'espace Hilbertien, Gauthier-Villars, Paris (1969). MR0352996 (50:5482)

[H] E.K. Haviland, On the moment problem for distribution functions in more than one dimension II, Amer. J. Math. 58 (1936), 164-168. MR1507139

[KM] S. Kuhlmann, M. Marshall, Positivity, sums of squares and the multidimensional moment problem, Trans. Amer. Math. Soc. 354 (2002), 4285-4301. MR1926876 (2003j:14078)

[KMS] S. Kuhlmann, M. Marshall, N. Schwartz, Positivity, sums of squares and the multidimensional moment problem II, Adv. Geom. 5 (2005), 583-606. MR2174483 (2006i:14064)

[PD] A. Prestel, C. N. Delzell, Positive polynomials, Springer, Berlin (2001). MR1829790 (2002k:13044)

[SCH] C. Scheiderer, Non-existence of degree bounds for weighted sums of squares representations, Journal of Complexity 21 (2005), 823-844. MR2182447 (2006k:14117)

[S1] K. Schmüdgen, Unbounded Operator Algebras and Representation Theory, Birkhäuser, Basel (1990). MR1056697 (91f:47062)

[S2] K. Schmüdgen, The K-moment problem for compact semi-algebraic sets, Math. Ann. 289 (1991), 203-206. MR1092173 (92b:44011)

[S3] K. Schmüdgen, On the moment problem of closed semi-algebraic sets, J. Reine Angew. Math. 558 (2003), 225-234. MR1979186 (2004e:47019)

Fachbereich Mathematik und Statistik, Universität Konstanz, 78457 Konstanz, GerMANY

E-mail address: tim.netzer@uni-konstanz.de 